\title{
NOTA SOBRE LA PRESENCIA DE HELIOTROPIUM KRAUSEANUM FEDDE (HELIOTROPIACEAE) EN CHILE
}

\section{NOTE ON THE PRESENCE OF HELIOTROPIUM KRAUSEANUM FEDDE (HELIOTROPIACEAE) IN CHILE}

\author{
Federico Luebert ${ }^{1} \&$ Raquel Pinto ${ }^{2}$ \\ 'Departamento de Silvicultura, Facultad de Ciencias Forestales, Universidad de Chile, Casilla 9206, Santiago, Chile. \\ Email: fluebert@uchile.cl \\ ${ }^{2}$ Equipo de Estudios de Ecosistemas de Niebla, Dalmacia 3251, Iquique, Chile.
}

\begin{abstract}
The presence of Heliotropium krauseanum Fedde is reported near Iquique in northern Chile $\left(19^{\circ} 37^{\prime} \mathrm{S}, 7^{\circ} 11^{\prime} \mathrm{O}\right)$. The species had been previously collected only in Perú. This report represents an important southward geographic range extension for the species, and a northward extension of the range of the section Cochranea of this genus in Chile.
\end{abstract}

Heliotropium krauseanum Fedde fue descrita en 1906 basándose en material proveniente de Mollendo (Departamento de Arequipa, Perú). Posteriormente ha sido colectada en diferentes localidades de Perú (Johnston 1928, Ferreyra 1961, Brako \& Zarucchi 1993, Arakaki \& Cano 2003, Galán de Mera et al. 2003, Weigend et al. 2003). Johnston (1928) señala su presencia en el norte de Chile, pero para una localidad indefinida ("Cobija, Iquiqui [sic] et Arica"), sobre la base de un ejemplar recolectado por Hugh Cuming y depositado en el Herbario Hooker, indicando que el mencionado ejemplar (Cuming 955, 1831) estaba etiquetado como proveniente de "Lima", lo que atribuye a "otro ejemplo del descuido y la imprecisión en las etiquetas de Hooker en sus últimos años" (Johnston 1928: 28). Marticorena \& Quezada (1985) se basaron en esta indicación para incluir la especie en el catálogo de la flora vascular de Chile, aunque en ausencia de colectas posteriores su presencia en Chile resultaba dudosa (C. Marticorena, comunicación personal). Recientemente Weigend et al. (2003) reportan el hallazgo de H. krauseanum en el Departamento de Lima, Perú, lo que hace suponer que la indicación de Johnston (1928) era errónea y la etiqueta del ejemplar de Cuming en el Herbario Hooker era correcta, confirmando a la especie como endémica de Perú y, por lo tanto, ausente en Chile.

Una colecta de $H$. krauseanum fue efectuada en octubre de 2002 por R. Pinto entre Caleta Junín y
Punta Pichalo, al sur de Pisagua, provincia de Iquique. Esta colecta pone otra vez en duda la indicación de Johnston (1928) y confirma la presencia de la especie en Chile.

La especie se presenta en un ecosistema de niebla, que se encuentra a $65 \mathrm{~km}$ en línea recta al norte de Iquique, en laderas de exposición sudoeste, en los altos del acantilado costero, sobre los $700 \mathrm{~m}$ en la Cordillera de la Costa. Es un sector de lomas suaves, suelos arenosos con cañadas poco profundas de roca triturada fina. La vegetación se desarrolla solamente durante la primavera de los años con presencia de eventos El Niño de gran intensidad.

Heliotropium krauseanum Fedde, in Just, Bot. Jahrb. 34: 72. 1908. Johnston, Contr. Gray Herb. 81: 28. 1928. Weigend et al., Arnaldoa 10: 62. 2003.

Holotipo: PERU, prope Mollendo, in saxosis, 50-100 $\mathrm{m}$, Weberbauer 1552 (B).

Heliotropium saxatile Krause, Bot. Jahrb. 37: 633. 1906. nom. illeg. non Heliotropium saxatile Brandeg. 1905.

Distribución geográfica: Acantilado costero (>700 m) de la provincia de Iquique en Chile, lomas costeras bajas $(<500 \mathrm{~m})$ de los departamentos de Arequipa, Moquegua y Tacna en Perú y zonas altas (1200-1750 m) de la provincia de Yauyos, departamento de Lima, Perú (Figura 1). 


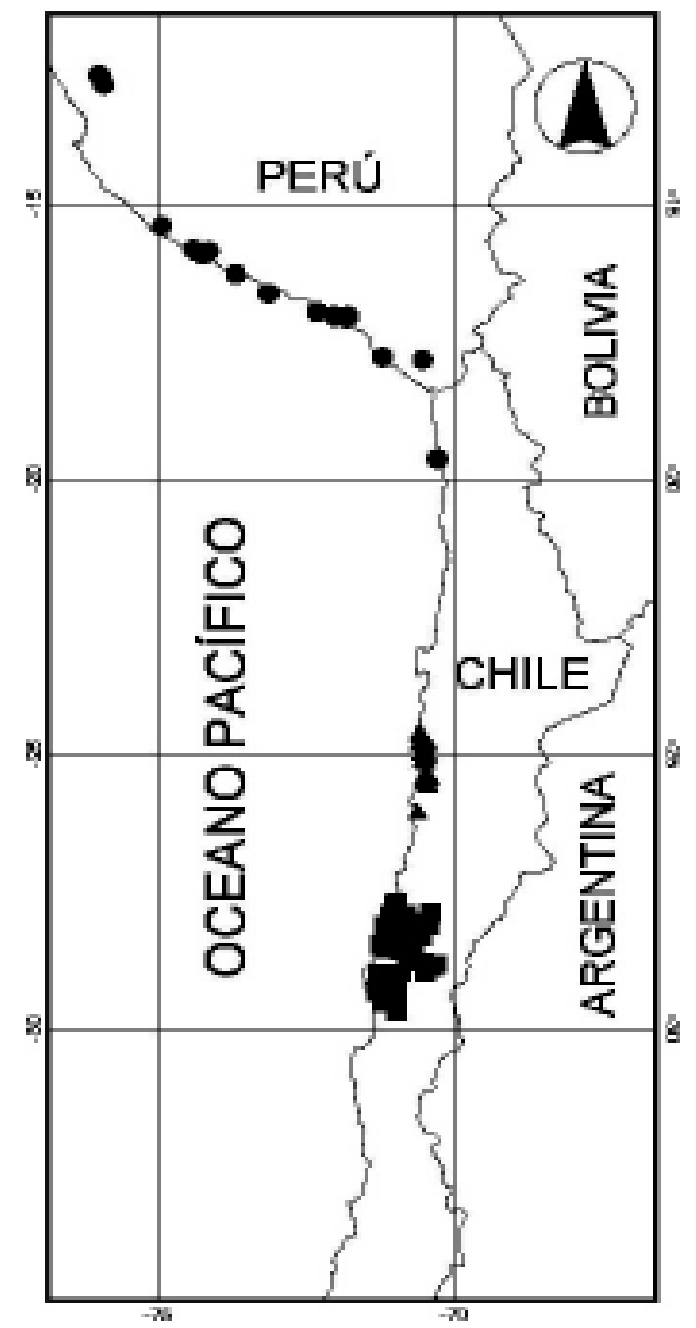

Figura 1. Mapa de distribución de Heliotropium krauseanum (círculos) y de las especies morfológicamente afines, $H$. taltalense (triángulos) y $H$. sinuatum (cuadrados)

\section{MATERIAL ESTUDIADO}

CHILE. I Región. Prov. Iquique. Entre Punta Pichalo y Caleta Junín, punta sur de Punta Pichalo, $19^{\circ} 37^{\prime} \mathrm{S}$, $70^{\circ} 11^{\prime} \mathrm{O}, 700 \mathrm{~m}, 22-\mathrm{IX}-2002$, R. PINTO 171 (SGO 151274).

La presencia de $H$. krauseanum en Chile constituye un nuevo límite sur para la especie, cuyo rango de distribución geográfica se extiende en cerca de $200 \mathrm{~km}$ y supone a su vez una ampliación hacia el norte del rango de distribución la sección Cochranea en Chile, aunque Johnston (1932: 7) ya había suge- rido la presencia de $H$. linariaefolium Phil., de la sección Cochranea, en las cercanías de Iquique, pero basándose en muestras muy incompletas y sin citar claramente el material.

De acuerdo con Johnston (1928), $H$. krauseanum, junto con H. sinuatum (Miers) Johnst. y H. taltalense (Phil.) Johnst. conforman un grupo disyunto (Figura 1) de especies morfológicamente afines dentro de la sección Cochranea. Estas especies se caracterizan por presentar hojas muy resinosas, sinuadas y con haz rugoso. Heliotropium taltalense y $H$. sinuatum fueron reunidas por Reiche (1907) bajo el nombre de H. rugosum Phil. (Fl. Atac. 38. 1860. nom. illeg. non H. rugosum Mart. et Gal. 1844). Por su parte, Johnston (1928) señala que $H$. krauseanum podría eventualmente corresponder a una variedad más septentrional y de flores más pequeñas de $H$. taltalense, cuyo estatus definitivo está todavía por aclarar. La siguiente clave, basada en Johnston (1928), permite diferenciar estas tres especies:

1. Hojas con nervios secundarios simples; plantas de la costa e interior de las provincias de Huasco, norte de Elqui y sur de Copiapó H. sinuatum 1. Hojas con nervios secundarios divididos; plantas de más al norte. ...2

2. Cáliz maduro 3-4 mm largo, densamente velloso; tubo corolar 3-4 mm largo; plantas de la costa del sur de la provincia de Antofagasta y norte de Chañaral H. taltalense

2. Cáliz maduro 2-3 mm largo, laxamente hirsuto; tubo corolar 2.5-3 mm largo; plantas de la costa de la provincia de Iquique (Chile) y del centro-sur de Perú

..H. krauseanum

La distribución de H. krauseanum conocida hasta ahora es continua en el centro de su rango geográfico (lomas costeras de los departamentos de Arequipa, Moquegua y Tacna en Perú), con poblaciones disyuntas en ambos extremos de su rango de distribución: Departamento de Lima, Perú (Weigend et al. 2003) y provincia de Iquique, Chile (Figura 1). Tal discontinuidad puede ser explicada por la falta de colectas en las áreas intermedias (departamento de Ica, Perú y provincia de Arica, Chile), o bien por ausencia de condiciones ecológicas apropiadas para la presencia de la especie. Es de notar que no se ha encontrado Heliotropium durante los recorridos realizados en 2002 (R. Pinto) en la costa de la provincia de Arica (Punta Madrid \& Camaraca). Dicho sector corresponde a una zona de máxima ari- 
dez en el gradiente latitudinal, producto del descenso de las precipitaciones (Almeyda 1950) y de la tendencia a una disminución de la incidencia de neblinas costeras hacia el norte de Chile (Cereceda \& Schemenauer 1991), que se manifiestan en una marcada discontinuidad de la vegetación (Muñoz-Schick et al. 2001, Fuentes 2003).

\section{AGRADECIMIENTOS}

Agradecemos a Mélica Muñoz por su buena disposición para la consulta del Herbario SGO y a Clodomiro Marticorena por sus valiosos comentarios.

\section{BIBLIOGRAFIA}

Almeyda, E. 1950. Pluviometría de las zonas del desierto y las estepas cálidas de Chile. Editorial Universitaria, Santiago. 162 pp.

Arakaki, M. \& A. CANo. 2003. Composición florística de la cuenca del río Ilo-Moquegua y Lomas de Ilo, Moquegua, Perú. Revista Peruana de Biología 10: 5-19.

BRAKo, I. \& J. ZARUCCHI. 1993. Catalogue of the Flowering Plants and Gymnosperms of Perú. Monographs in Systematic Botany from the Missouri Botanical Garden 45: 1-1286.
Cereceda, P. \& R. Schemenauer. 1991. The occurrence of fog in Chile. Journal of Applied Meteorology 30: 1097-1105.

Ferreyra, R. 1961. Las lomas costaneras del extremo sur del Perú. Boletín de la Sociedad Argentina de Botánica 9: 87-120.

Fuentes, R. 2003. Vegetación arbustiva y herbácea de Tarapacá. Guías de Identificación y Biodiversidad Chilena. Universidad Arturo Prat, Iquique. $11 \mathrm{pp}$.

Galán De Mera, A., C. Cáceres \& A. González. 2003. Nuevas adiciones a la flora del Perú, III. Candollea 58: 325-337.

Johnston, I.M. 1928. Studies in the Boraginaceae VII. The South American Species of Heliotropium. Contributions from the Gray Herbarium 81: 3-73.

Johnston, I.M. 1932. New records for the flora of the Nitrate Coast. Revista Chilena de Historia Natural 36: 4-8.

Marticorena, C. \& M. Quezada. 1985. Catálogo de la flora vascular de Chile. Gayana Botanica 42: 1157.

Muñoz-Schick, M., R. Pinto, A. Mesa \& A. MoreiraMuÑoz. 2001. "Oasis de neblina" en los cerros costeros del sur de Iquique, región de Tarapacá, Chile, durante el evento El Niño 1997-1998. Revista Chilena de Historia Natural 74: 389-405.

Reiche, K. 1907. Estudios críticos sobre la flora de Chile. Anales de la Universidad de Chile 121: 227-250.

Weigend, M., G. Brokamp, A. Kirbach \& H. Förther. 2003. Notas sobre Heliotropium krauseanum Fedde, la única especie de Heliotropium sect. Cochranea del Perú (Heliotropiaceae = Boraginaceae subfam. Heliotropioideae). Arnaldoa 10: 61-74.

Fecha de recepción: 26.04.04

Fecha de aceptación: 14.06.04 